Ann. Biol. anim. Bioch. Biophys., I97I, 11 (3), 379-387.

\title{
ETUDE DU DÉVELOPPEMENT IN VITRO DES BLASTOCYSTES DE LAPIN
}

\author{
Suzanne WINTENBERGER-TORRÈS et Marie-Christine DANEL \\ avec la collaboration technique de Nicole VermeIre
}

Station centrale de Physiologie animale, Centre national de Recherches zootechniques, I. N.R. A., 78 - Jouy-en-Josas

\section{RÉSUMÉ}

On a étudié la croissance des blastocystes de Lapine âgés de 5 jours dans du I99 modifié ou du sérum de Veau fotal. On a comparé les résultats obtenus quand les cultures sont faites dans des tubes de verre avec $0,5 \mathrm{ml}$ de milieu, des salières ou des boîtes Falcon contenant $\mathrm{I}, 5 \mathrm{ml}$ de milieu sous atmosphère d'air +5 p. Ioo de $\mathrm{CO}_{2}$.

Les expériences poursuivies pendant 8 heures ont montré que, dans le sérum de Veau, la croissance est meilleure en salière qu'en tube. Les résultats ne sont pas significativement différents entre salières et boîtes Falcon.

Le sérum de veau assure une croissance significativement meilleure que le I99.

On observe une chute de croissance générale après les 4 premières heures de culture, quelles que soient les conditions de milieu, sauf pour les plus gros blastocystes.

Les plus petits blastocystes ont une vitesse de croissance inférieure aux plus gros et cette différence ne fait qu'augmenter avec le temps de culture.

Des différences de taille étant observées naturellement chez des blastocytes issus d'une même ponte, la désynchronisation de leur développement pose le problème de la survie des plus petits après l'implantation.

La culture in vitro de l'œuf fécondé de Souris jusqu'au stade blastocyste, dans des milieux entièrement synthétiques, a permis l'étude de son métabolisme. Les blastocystes ainsi obtenus peuvent se développer normalement puisque leur transplantation dans des femelles receveuses a donné de 20,4 p. IOo (MCL,AREN et BIGGERS, I958) à 29,4 p. Ioo de jeunes (BowMaN et MCLAREN, I970). Cependant, ces pourcentages sont relativement faibles, et ADAMs (I970) observe que la prolongation du temps de culture de morulas de Lapine de 24,48 et 72 heures s'accompagne d'une diminution du nombre de jeunes nés respectivement $74,6,48,3$ et 20,5 p. Ioo.

Le milieu de culture ne satisfait done pas entièrement les besoins exigés au début du développement embryonnaire ; de fait, les blastocystes de Souris récupérés après culture sont de taille inférieure à ceux d'âge correspondant développés in utero. 
Le rythme de segmentation est de $\mathrm{I}$ division toutes les Io heures in vivo et une toutes les 14 heures in vitro (Bowman et MCLAREN, I970).

Adams (1970) a également enregistré des retards de segmentation d'œufs de Lapine cultivés in vitro et pour faire coïncider aussi étroitement que possible l'âge des œufs cultivés transplantés, au stade physiologique correspondant de l'utérus receveur, il a été amené à accoupler les femelles receveuses après les femelles donneuses.

Le ralentissement de la segmentation observé in vitro pourrait être responsable du faible développement embryonnaire. C'est ce que paraît indiquer l'expérience de GATES (I965) : les œufs de Souris imbred (BALB/C $\times$ BALB/C) qui se développent plus lentement que les œufs hybrides (BALB/C $\times$ r29) donnent un pourcentage de jeunes nés de I7 p. IOO contre 50 p. IOO.

Ces résultats font ressortir l'importance qu'on doit attacher aux conditions de culture in vitro quand on veut se livrer à des études métaboliques ou à des transplantations.

La croissance en culture semble être un bon critère pour apprécier la valeur d'une technique de culture.

GaRDNER et EDWARDS (I968) ont montré que, pour les œufs de Lapine, le sérum de Veau fotal assure une meilleure croissance que le $\mathrm{F}_{10}$ modifié utilisé par STAPIES (rg67).

C'est pourquoi nous avons entrepris une étude de la croissance des blastocystes de Lapine âgés de 5 jours dans des conditions variées de milieux.

\section{MATÉRIEL ET MÉTHODES}

Les blastocystes sont prélevés sur des Lapines Néo-Zélandaises et Petit-Russe, abattues 5 jours $\mathrm{I} / 2$ post coïtum. On perfuse directement avec le milieu de culture dans une étuve à $37^{\circ} \mathrm{C}$ La culture se poursuit dans un caisson, situé à l'intérieur de l'étuve, où circule de l'air $+5 \mathrm{p}$. Ioo $\mathrm{CO}_{2}$. Au début de la mise en culture le $\mathrm{pH}$ peut varier de 6,8 à $7, \mathrm{I}$ selon le milieu utilisé. En fin d'expérience l'écart n'a jamais dépassé 3/ro pour un même milieu et sur l'ensemble des mesures les variations ont été comprises entre 6,7 et 7,2 .

Les résultats de GARDNER et EDWARDs étant en faveur du sérum de Veau, nous avons tout d'abord étudié l'influence du volume liquide et du mode de culture avec ce milieu.

\section{a) Culture en tube}

Les blastocytes, dont le volume peut varier de 5 à $\mathrm{I}_{4} \mathrm{~mm}^{3}$ sont introduits avec environ $0,5 \mathrm{ml}$ de milieu, dans un tube de verre fermé aux deux extrémités par de l'huile de paraffine. Une bulle d'air est emprisonnée de chaque côté, entre le milieu et l'huile. Plusieurs tubes sont fixés dans un rotor à révolution discontinue pour faciliter les échanges entre le blastocyste et le milieu. Le tout est placé dans le caisson.

\section{b) Culture en salière}

Chaque blastocyste est placé dans une salière avec I,5 $\mathrm{ml}$ de milieu, un film d'huile de paraffine lute le couvercle et le rebord de la salière.

\section{c) Culture en boitte Falcon} central.

Les boîtes utilisées ont $5,5 \mathrm{~cm}$ de diamètre et contiennent $\mathrm{I}, 5 \mathrm{ml}$ de milieu dans l'anneau 
d) Milieux

Milieu 1: il est composé de 75 parties de 199 (Difco)

+ ro parties de sérum de Lapin (chauffé $30 \mathrm{mn}$ à $5^{\circ} \mathrm{C}$ )

+5 parties de sérum de Poulain

+ ro parties de tampon phosphate ( $\mathrm{pH} 7)$

+ Ioo $\mu \mathrm{g} / \mathrm{ml}$ de pénicilline et $50 \mu \mathrm{g} / \mathrm{ml}$ de streptomycine.

Sa pression osmotique est de $284,5 \mathrm{~m}$ Osm.

Milieu 2: il comprend 98 parties de sérum de Veau fœtal frais ou lyophilisé et 2 parties de tampon phosphate ( $\mathrm{pH} 7$ )

+ Iоo $\mu \mathrm{g} / \mathrm{ml}$ de pénicilline. Sa pression osmotique est de $299 \mathrm{mOsm}$.

On mesure les blastocystes en faisant la moyenne de deux diamètres perpendiculaires, au début de la mise en culture et après 2, 4, 6 ou 8 heures de culture.

\section{RÉSULTATS}

Cette première série d'expériences a été réalisée en prenant comme milieu de culture du sérum de Veau. On a comparé deux à deux les croissances après séjour de 6 heures dans des tubes ( 54 blastocystes) et des salières (68 blastocystes); dans des salières (50 blastocystes) et des boîtes Falcon (3I blastocystes).

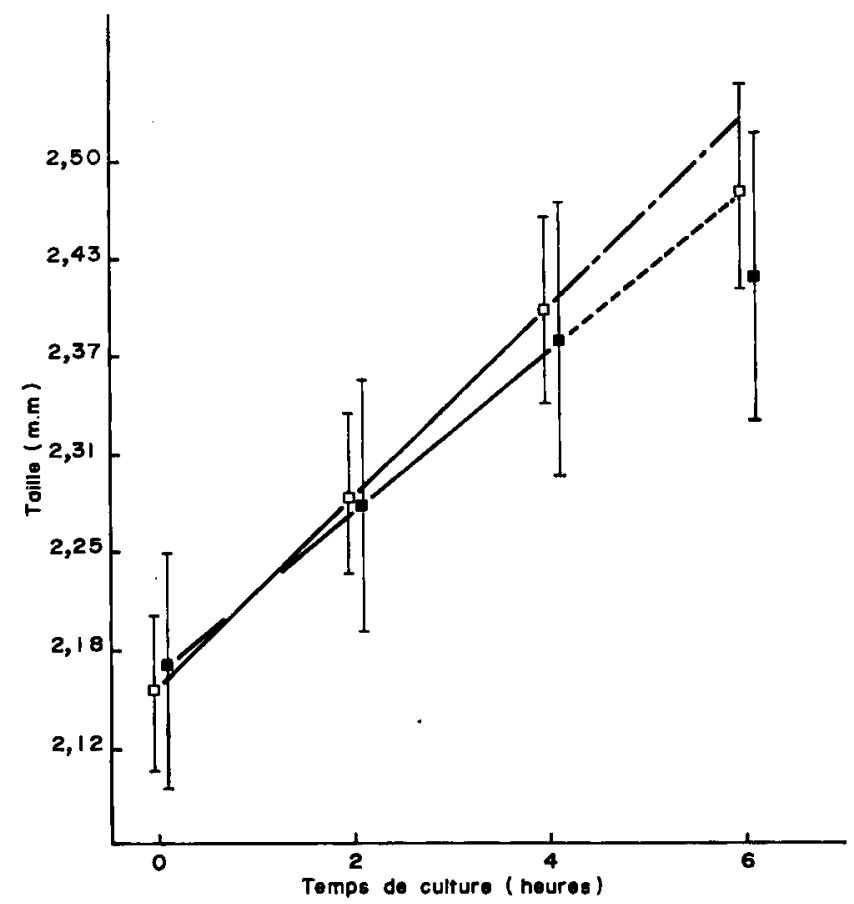

FIG. I. - Comparaison des croissances en tubes et en salieres

ㅁalières $\quad b=0,060$

tubes $\quad b=0,054$
droites calculées pour l'intervalle de temps $t_{0}-t_{4}$ 


\section{a) Comparaison tubes-salières}

La taille moyenne augmente en fonction du temps (fig. I) pendant 6 heures. Mais l'ajustement linéaire de la droite de régression aux points moyens n'est possible que pour les temps 0,2 et 4 heures; pendant les deux dernières heures, il y a une diminution de la vitesse de croissance.

Les coefficients des droites (de o à 4 heures) qui sont respectivement pour les salières de $b=0,0602$ et pour les tubes $b=0,054 \mathrm{I}$ ne sont pas significativement différents.

Par contre, la variabilité des réponses est significativement plus grande lors de la culture en tube (importance comparée des écarts-types). On retrouve aussi un pourcentage plus important de blastocystes qui ont une croissance régulière (sans arrêt ni chute) dans le cas des salières ( 85 p. Ioo) que dans les tubes ( 56 p. xoo). La différence de ces pourcentages est hautement significative.

A la fin des 6 heures de culture, on retrouve 4,4 p. Ioo seulement de blastocystes rétractés en salières contre $I_{7} \mathrm{p}$. Ioo en tubes.

\section{b) Comparaison salières boîtes Falcon}

Les mesures ont été prises uniquement aux temps 0,4 et 8 heures. Quelle que soit la technique de culture employée on retrouve une chute de croissance entre 4 et 8 heures (fig. 2).

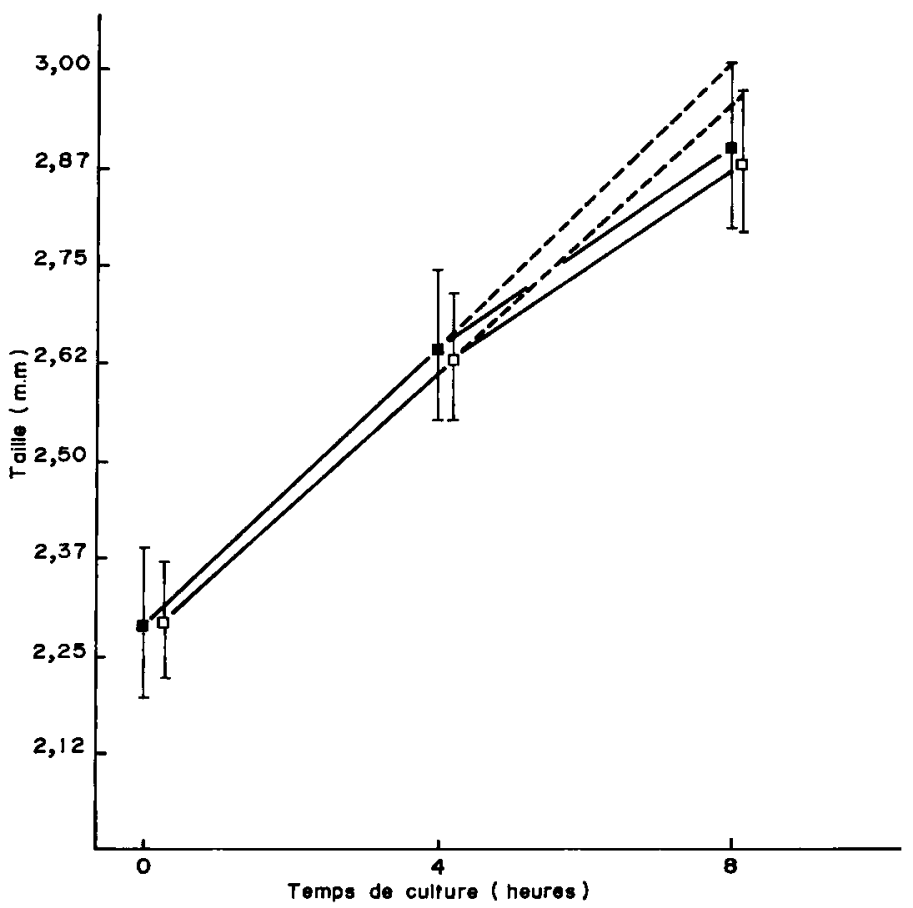

Frg. 2. - Comparaison des croissances en salières et en bottes Falcon

$$
\begin{aligned}
& \text { - boîte } \\
& \square \text { salière }
\end{aligned}
$$


On constate une bonne homogénéité des tailles moyennes pour les deux séries expérimentales. La croissance est légèrement supérieure pour les blastocystes cultivés en boîtes, mais la différence avec les salières n'est pas significative.

\section{c) Étude de la croissance entre 0 et 8 heures}

La constance des résultats obtenus en salières nous a incitées à choisir ce conditionnement pour préciser la durée du ralentissement de croissance enregistré après 4 heures de culture.

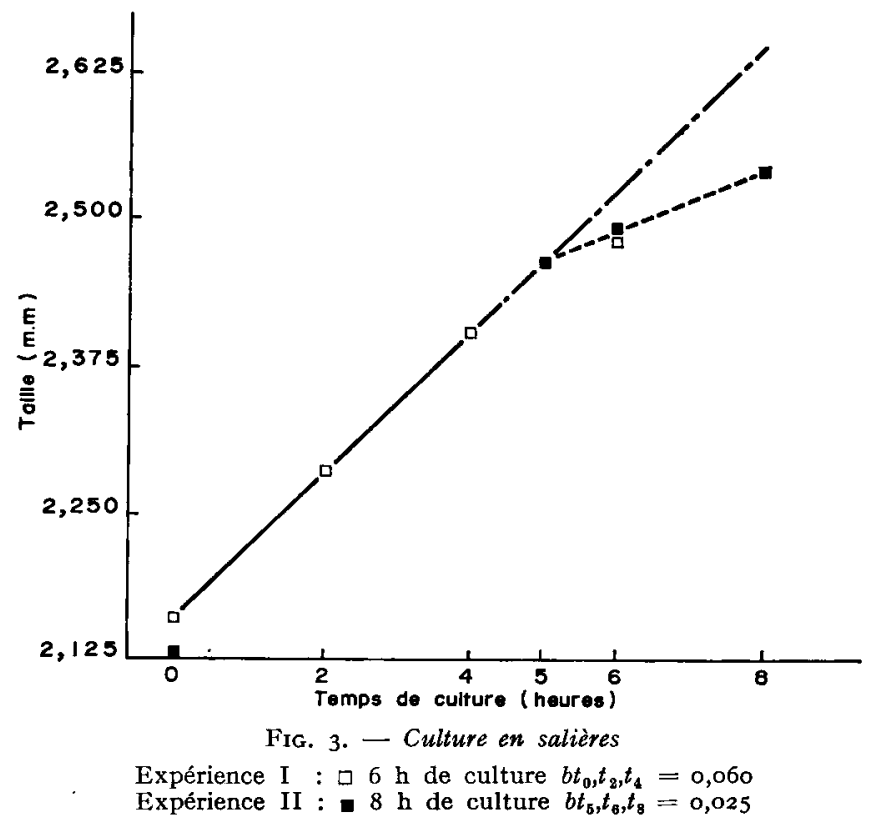

66 blastocystes faisant l'objet d'une autre expérience (fig. 3) ont été cultivés pendant 8 heures en mesurant leur taille à o, 5,6 et 8 heures, les temps 5 et 6 heures ayant été choisis pour déterminer plus précisément le moment où la croissance se ralentit. La croissance n'est pas linéaire entre o et 8 heures. Elle l'est entre o et 4 heures (expérience I) et également entre 5 et 8 heures, mais les pentes sont significativement différentes : 0,060 entre o et 4 heures; 0,025 entre 5 et 8 heures.

Ces résultats montrent que la courbe s'infléchit après 5 heures de culture : la vitesse de croissance diminuant de plus de moitié.

Bien que les expériences I et II (fig. 3) n'aient pas été faites en même temps et qu'il s'agisse de deux populations différentes de blastocystes, les résultats sont très comparables, car les tailles moyennes des deux populations au temps o ne sont pas différentes. Le point de rupture à $t_{5}$ dans l'expérience II se place exactement sur le prolongement de la droite de régression de l'expérience $I$; de plus les tailles moyennes après 6 heures de culture ne sont pas significativement différentes. 


\section{d) Comparaison des milieux de culture}

Cette expérience a été effectuée dans des salières en utilisant 33 blastocystes pour le Milieu I (I99) et 68 pour le Milieu II (sérum de Veau).

La croissance des blastocystes n'est pas linéaire pendant les 6 heures de culture. Les écarts de linéarité sont négligeables lorsqu'on ne s'intéresse qu'aux quatre premières heures (fig. 4).

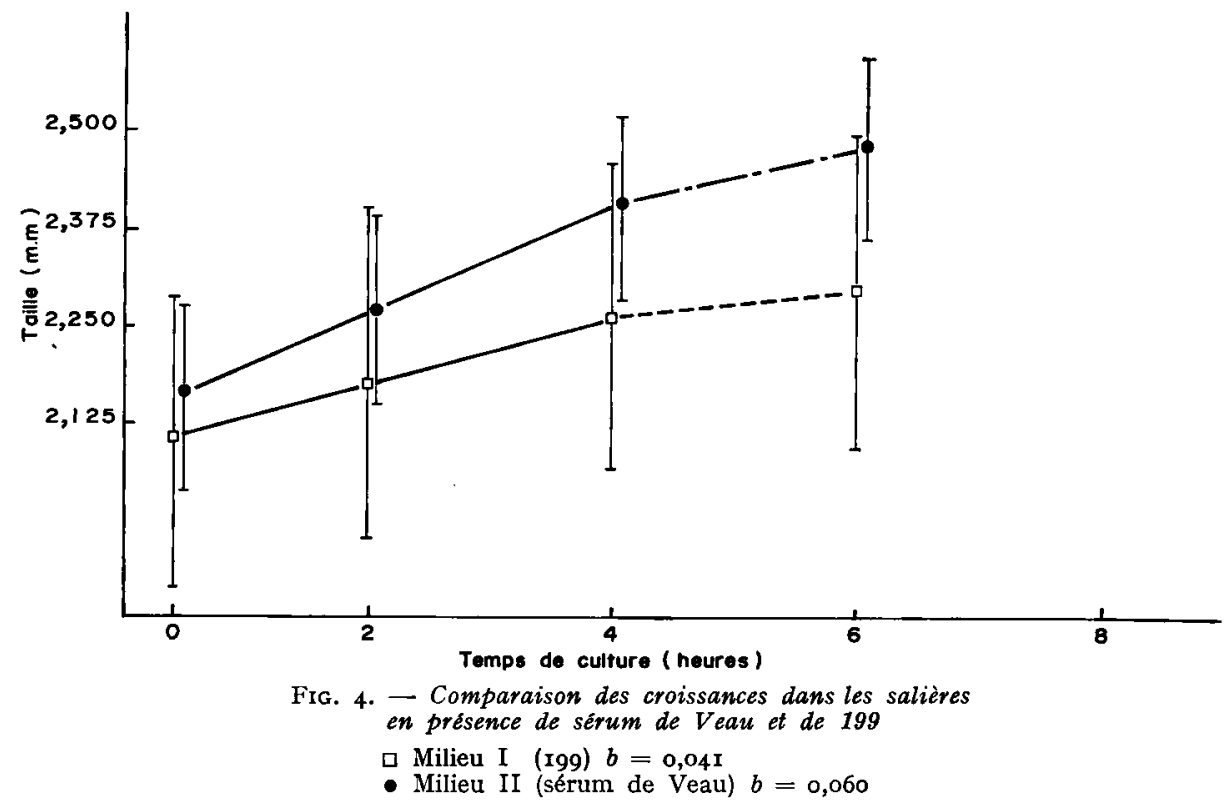

La différence des coefficients des droites de régression est hautement significative :

$$
\begin{aligned}
& b=0,04 \text { I Milieu I (I99) } \\
& b=0,06 \text { o Milieu II (sérum de Veau) }
\end{aligned}
$$

Les dispersions sont légèrement plus importantes dans le Milieu I que dans le Milieu II. Mais elles restent pratiquement semblables pendant la durée de l'expérience.

\section{e) Infuence de la taille des blastocystes sur leur développement en culture}

La différence de taille des blastocystes constatée à la perfusion nous a incitées à examiner leur comportement en cours de culture en fonction de leur taille initiale. Les blastocystes cultivés en boîtes Falcon et en salières dans du sérum de Veau ont été divisés en 6 classes de tailles; on a calculé la moyenne de croissance pour chacun d'eux en distinguant la période 0-4 heures et 4-8 heures (fig. 5).

Pendant la première période, seuls les blastocystes dont la taille à $t_{0}$ est comprise 
entre $\mathrm{I}, 25$ et $\mathrm{I}, 56 \mathrm{~mm}$ ont une croissance faible. Pour les tailles supérieures comprises entre $\mathrm{I}, 56$ et $3, \mathrm{I} 2 \mathrm{~mm}$ la croissance est voisine de $0,3 \mathrm{~mm}$.

Pendant la seconde période, de 4 à 8 heures, l'influence de la taille est encore accentuée : seuls les blastocystes compris entre $2,8 \mathrm{I}$ et $3, \mathrm{I} 2 \mathrm{~mm}$ ont en moyenne une croissance égale à celle qu'ils avaient précédemment. Il semble donc que les plus gros blastocystes ne subissent pas un ralentissement de croissance après 4 heures de séjour dans le sérum de Veau, ce que ne laissaient pas apparaître les moyennes de croissances calculées sur l'ensemble des blastocystes sans tenir compte de leur taille initiale.

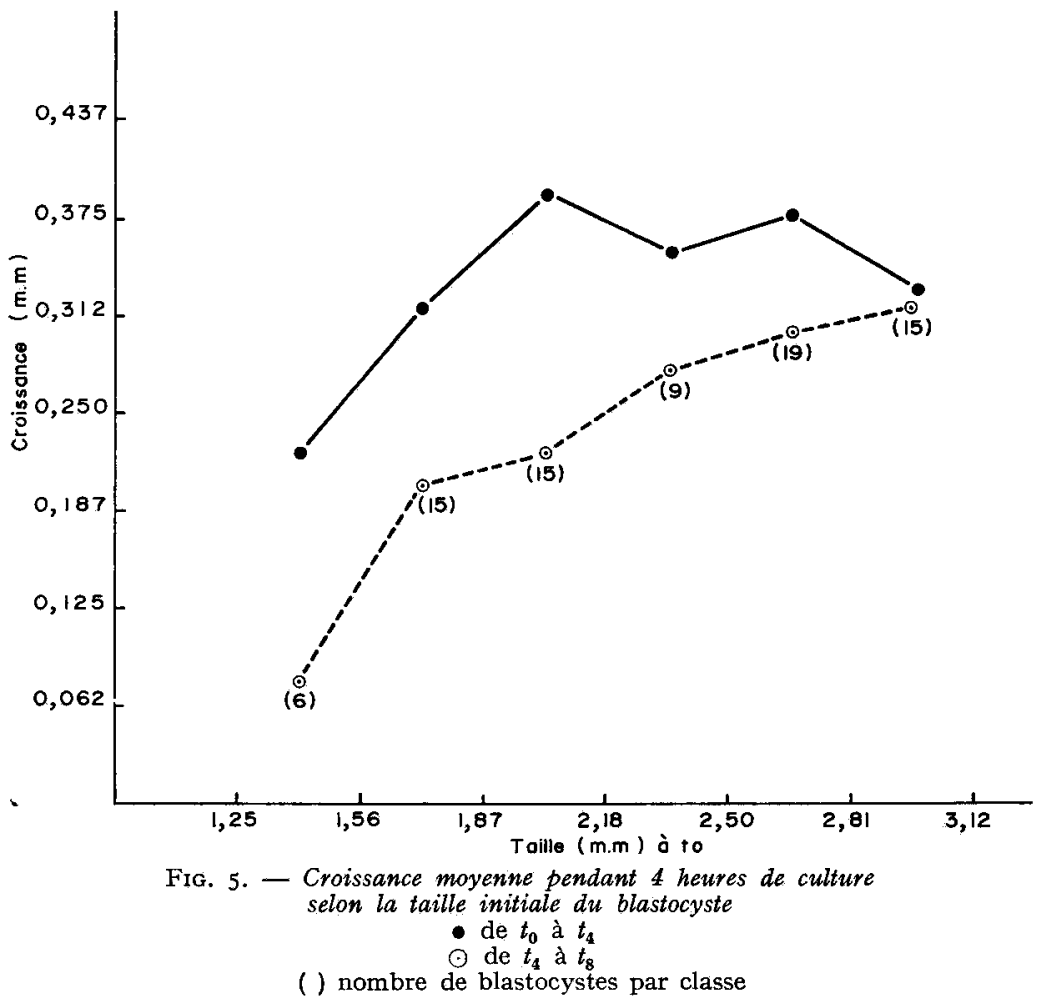

\section{CONCLUSION}

Quel que soit le mode de culture utilisé, il apparait que le rythme de croissance enregistré pendant les 4 premières heures ne peut être qu'exceptionnellement conservé plus longtemps. Smith (I970), dans du I99, constate le même phénomène avec des blastocystes de Lapines prélevés 6 jours p. $\mathrm{c}$.

La culture d'organes montre que le rejet dans le milieu de culture des produits du métabolisme entraîne une rapide inhibition de la croissance. On pourrait imaginer qu'un tel phénomène est à l'origine de la chute de croissance après 4 heures. Mais le fait que ce soient les plus gros blastocystes qui conservent le meilleur coefficient de croissance est en défaveur de cette hypothèse. 
L'épuisement du milieu quand le temps de culture se prolonge ne peut pas être évoqué non plus, puisque cela impliquerait un ralentissement de croissance plus rapide pour les gros blastocystes.

La période critique correspond à un ralentissement de la croissance qui concerne la multiplication des cellules trophoblastiques. On a pu vérifier en effet (WINTENBERGER-TORRÈS, I970) que chez des blastocystes prélevés après des temps variables de culture, il y a une corrélation étroite entre la taille et le nombre de cellules.

Les blastocystes ne sont pas indifférents au mode de culture utilisé. La fragilité constatée dans les tubes tient peut-être à l'insuffisance du volume liquide mis à leur disposition, hypothèse que la similitude des résultats obtenus en boîtes Falcon et en salière semble confirmer.

Dans les meilleures conditions de culture que nous avons réalisées, il apparaît que la croissance n'est pas constante pour tous les blastocystes. Pendant le début de la culture, les petits blastocystes ont toujours une croissance inférieure aux autres. L'hétérogénéité des tailles des blastocystes constatée au $5^{\mathrm{e}}$ jour ne fait donc que s'accentuer au cours de leur développement et pose le problème de la survie des plus petits d'entre eux. Il est en effet probable que le retard accumulé tend à les désynchroniser du stade physiologique de l'utérus où ils se développent.

La difficulté de maintenir in vitro le rythme de croissance normal des blastocystes expliquerait la forte proportion d'échecs enregistrés après transplantation par McLaren chez la Souris et par Adams chez la Lapine.

Rę̧u pour publication en mars 1971.

\author{
SUMMARY \\ AN INVESTIGATION INTO THE IN VITRO DEVELOPMENT \\ OF RABBIT BLASTOCYSTS
}

Investigations were carried out on the development of 5 days rabbit blastocysts in modified TC 199 or calf serum media, and experiments lasting for $8 \mathrm{hrs}$ were ddesigned to compare thefollo, wing culture techniques : glass tube $(0,5 \mathrm{ml})$ in rolling system, watchglass and Falcon dish $(\mathrm{I}, 5 \mathrm{ml})$ gassed with $\mathrm{CO}_{2}$ : air mixture $(5: 95)$.

Growth rate of blastocysts in calf serum medium was found to be higher with watchglass than with glasstubes. There was no significant difference between watchglass and Falcon dish.

Growth rate was significantly higher in calf serum than in TC 199.

A decrease in the growth rates of all blastocysts except the biggest was noticed after the firist 4 hrs of culture, irrespective of the medium or technique. The growth rate of smaller-sized blastocysts was lower than that of the bigger-sized, and this difference tended to increase with the duration of culture. The deviation observed in vitro of growth rates of smaller vs-bigger blastocysts leads us to question the survival in vivo of the smaller blastocysts.

\title{
RÉFÉRENCES BIBLIOGRAPHIQUES
}

Adams C. E., I970. The development of rabbit eggs after culture in vitro for I*4 days. J. Embryol. exp. Morph., 28, 21-34.

Bowman P., MaC LAREN A., 197o. Viability and growth of mouse embryos after in vitro culture and fusion. J. Embryol. exp. Morph., 28, 693-704. 
Gardner R. L., Edwards R. G., 1968. Control of the sex ratio at full term in the rabbit by transferring sexed blastocysts. Nature, 218, 346-348.

Gates A. H., r965. Rate of ovular development as a factor in embryonic survival. In Preimplariation Stages of Pregnancy. Ciba Found. Symp.

Mac Laren A., Biggers J. D., 1958. Successful development and birth of mice cultivated in vitro as early embryos. Nature, $182,877-878$.

NEw D. A. T., 1966. Development of rat embryos cultured in blood area. J. Reprod. Fert., 12, 504524.

New D. A. T., Daniel J. C., 1969. Cultivation of rat embryos explanted at 7,5 to 8,5 days of gestation. Nature, 223, 5I5-5I6.

New D. A. T., Coppola P. T., I97o. Effects of different oxygen concentrations on the development of rat embryos in culture. J. Reprod. Fert., 21, Iog-I 18.

Sмгтн M. W., I97o. Active transport in the rabbit blastocyst. Experientia, 26, 736-738.

Staples R. E., r967. Development of 5 days rabbit blastocysts after culture at $37^{\circ} \mathrm{C}$. J. Reprod. Fert., 13, 369-372.

Wintenberger-Torrès S., I970. Étude de la respiration des blastocytes de Lapine en culture in vitro. Ann. Biol, anim. Bioch. Biophys., 10, 187-194. 